Research Paper

\title{
Arnicolide D Inhibits Triple Negative Breast Cancer Cell Proliferation by Suppression of Akt/mTOR and STAT3 Signaling Pathways
}

\author{
Zhao Qu ${ }^{1}$, Yushan Lin ${ }^{1}$, Daniel Kam-Wah Mok ${ }^{1,2}$, Qingya Bian ${ }^{3}$, William Chi-Shing Tai ${ }^{1,2}{ }^{\varpi}$, Sibao Chen ${ }^{1,2,3} \bowtie$ \\ 1. State Key Laboratory of Chinese Medicine and Molecular Pharmacology (Incubation), Shenzhen Research Institute, The Hong Kong Polytechnic University, \\ Shenzhen 518057, China. \\ 2. Department of Applied Biology \& Chemical Technology, The Hong Kong Polytechnic University, Hung Hom, Hong Kong S.A.R., China. \\ 3. Institute of Medicinal Plant Development, Chinese Academy of Medical Sciences \& Peking Union Medical College, Beijing 100193, China. \\ $\triangle$ Corresponding authors: William Chi-Shing Tai, E-mail: william-cs.tai@polyu.edu.hk; Tel: +852-3400-8722; Sibao Chen, E-mail: sibao.chen@polyu.edu.hk; Tel: \\ +86-0755-26737182; Fax: +86-0755-26972852.
}

(1) The author(s). This is an open access article distributed under the terms of the Creative Commons Attribution License (https://creativecommons.org/licenses/by/4.0/). See http://ivyspring.com/terms for full terms and conditions.

Received: 2020.04.12; Accepted: 2020.05.29; Published: 2020.06.15

\begin{abstract}
Triple-Negative Breast Cancer (TNBC) is a most dangerous breast cancer subtype. The naturally occurring sesquiterpene lactone, arnicolide $D(A D)$, has proven effective against a variety of tumors, however, the inhibitory effects of $A D$ against TNBC and the underlying mechanisms remain unclear. In the present study, two TNBC cell lines (MDA-MB-231 and MDA-MB-468) and an MDA-MB-231 xenograft mouse model were employed to investigate the anti-TNBC effects of $A D$ in vitro and in vivo. Cell viability was assessed by MTT assay. Cell cycle arrest and apoptosis were analyzed by flow cytometry. Protein levels were determined by immunoblotting. In vitro studies demonstrated that AD significantly decreased cell viability, and induced G2/M cell cycle arrest and apoptosis. In vivo assays showed that oral administration of 25 or $50 \mathrm{mg} / \mathrm{kg}$ AD for 22 days led to a reduction of tumor weights by $24.7 \%$ or $41.0 \%$, without appreciable side effects. Mechanistically, AD inhibited the activation of Akt/mTOR and STAT3 signaling pathways. Based on our findings, $A D$ is a promising candidate for development as an adjunctive therapeutic drug for TNBC.
\end{abstract}

Key words: Arnicolide D; Triple negative breast cancer; Akt/mTOR; STAT3

\section{Introduction}

Breast cancer is the most common malignancy in women worldwide. According to the World Health Organization International Agency for Research on Cancer, there were approximately 2.1 million newly diagnosed female breast cancer cases in 2018, accounting for around $25 \%$ cancer cases among women worldwide [1]. Triple-Negative Breast Cancer (TNBC) is characterized by a lack of the estrogen receptor (ER), progesterone receptor (PR) and human epidermal growth receptor 2 (HER-2) that are commonly found in breast cancer, and accounts for approximately $15 \%-20 \%$ of all breast cancer cases [2]. TNBC tumors do not respond to endocrine or anti-HER2 therapies, and the systemic treatment is limited to cytotoxic chemotherapy [3]. Furthermore, TNBC is associated with a high risk of relapse and metastasis, short progression-free survival, and a lower overall survival when compared to other breast cancer subtypes [4]. Therefore, novel therapeutic drugs are necessary to combat TNBC.

Natural products are an important source of drugs due to their abundance and high chemical structural diversity. Increasing amounts of research have shown that a variety of natural products can suppress proliferation of TNBC cells by blocking the cell cycle, inducing apoptosis, and inhibiting angiogenesis and migration [5-8]. Thus, natural products are attracting increasing amounts of attention from researchers in the cancer field. Arnicolide D (AD) (Figure 1), a natural sesquiterpene lactone extracted from Centipeda minima [9], has been reported to exhibit anti-bacterial [9] and anti-cancer 
effects, in cancers including colon carcinoma [10], melanoma [11], and nasopharyngeal carcinoma [12]. However, there have been no reports on the effects of $\mathrm{AD}$ in TNBC. In this study, we aimed to investigate the anti-cancer effects of AD in TNBC. In addition, the underlying molecular mechanisms were also explored.

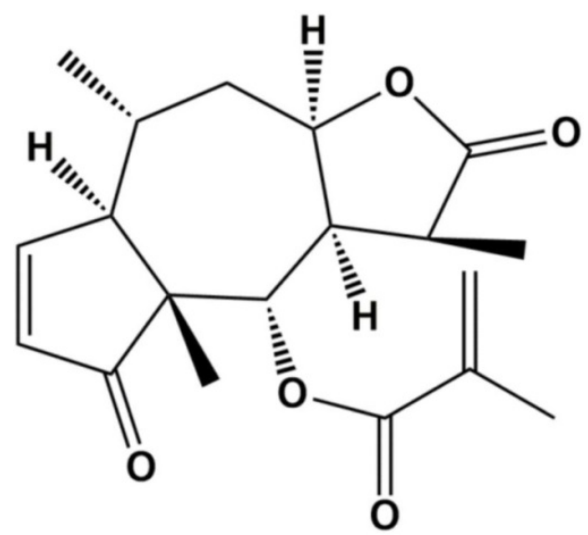

Figure 1.Chemical structure of Arnicolide D.

\section{Materials and Methods}

\section{Materials}

Arnicolide D $\left(\mathrm{C}_{19} \mathrm{H}_{24} \mathrm{O}_{5}, \mathrm{CAS34532-68-8)}\right.$ was purchased from Jiangsu Yongjian Pharmaceutical Co., Ltd. (Jiangsu, China). The purity of Arnicolide D was over $98 \%$ as analysed by high-performance liquid chromatography.

\section{Cell culture}

TNBC cell lines MDA-MB-231 and MDA-MB-468 and the non-TNBC cell line MCF7 were purchased from the American Type Culture Collection (ATCC, Manassas, VA, USA), and maintained in DMEM containing $10 \%$ heat-inactivated $\mathrm{FBS}$ in a $37^{\circ} \mathrm{C}, 5 \%$ $\mathrm{CO}_{2}$ and humidified incubator. All cell lines were used within 20 passages and 2 months after resuscitation, and mycoplasma testing was routinely performed using the PCR Mycoplasma Detection Kit (TransGen Biotech, Beijing, China).

\section{Cell viability assay}

To investigate the inhibitory effects of $\mathrm{AD}$ on breast cancer cell viability, $5 \times 10^{3}$ (MDA-MB-231 and MCF-7) or $1 \times 10^{4}$ (MDA-MB-468) cells were seeded into a 96-well plate and incubated for $24 \mathrm{~h}$. Cells were then exposed to different concentrations of AD for 24, 48 or $72 \mathrm{~h}$. Cells without treatment were used as a control. Cell viability was measured using MTT (Sigma-Aldrich), following manufacturer's protocols. $\mathrm{IC}_{50}$ values of $\mathrm{AD}$ were used to evaluate the in vitro anti-cancer cytotoxicity.

\section{Cell cycle and apoptosis analysis by flow cytometry}

MDA-MB-231 and MDA-MB-468 cells were seeded in 6-well plates and treated with AD for 24 or $48 \mathrm{~h}$. Cell pellets were collected and centrifuged at $1000 \mathrm{rpm}$ for $5 \mathrm{~min}$. For the cell cycle analysis, cells were fixed with ice-cold $70 \%$ ethanol at $4^{\circ} \mathrm{C}$ overnight, then stained with PI (Beyotime, Shanghai, China) and measured using a BD Accuri C6 flow cytometry system (Becton Dickson Immunocytometry-Systems, San Jose, CA, USA) and the data analyzed by ModFit software (ModFit LT 5.0, Verity Software House, Inc., Topsham, ME, USA). For apoptosis analysis, cell pellets were stained with FITC-labeled Annexin V and PI and assessed immediately using a CytoFLEX flow cytometer (Beckman Coulter, Brea, CA, USA) analysis immediately.

\section{Hoechst 33342 staining}

Briefly, the cells were treated with AD for $24 \mathrm{~h}$. Following washes with PBS, cells were fixed with $4 \%$ paraformaldehyde, stained with Hoechst 33342 for 15 min at room temperature, and then examined by fluorescence microscopy (Nikon, Tokyo, Japan).

\section{Wound-healing assay}

Cell migration was evaluated by wound-healing assay. MDA-MB-231 cells $\left(4 \times 10^{4}\right.$ cells/well $)$ were seeded into $35 \mathrm{~mm}$ high $\mu$-dishes with culture inserts (ibidi, Germany). Following removal of culture inserts and washes with medium, cells were treated with various concentrations of $\mathrm{AD}$, or batimastat (Aladdin, Shanghai, China) as a positive control. Migration of cells into the wound area was photographed under a microscope at 0, 6, 12, 24 and $48 \mathrm{~h}$ time points. The scratch open area was calculated using ImageJ software.

\section{Western blot assay}

Total protein was extracted using cell lysis RIPA buffer (Cell signaling technology, Beverly, MA, USA) and protein concentrations were determined by BCA Protein Assay (Thermo Scientific). Protein samples (10 $\mu \mathrm{g}$ per well) were subjected to $10 \%-15 \%$ SDS-PAGE and then wet-transferred to PVDF membranes. The blots were then blocked in 5\% non-fat milk and incubated with primary antibodies in 5\% BSA overnight. HRP-conjugated secondary antibodies were used following washes with TBST buffer. Protein bands were detected using ECL western blotting substrate (Tanon, Shanghai, China). QuantityOne software (Bio-Rad, Hercules, California, USA) was used for band density analysis. 


\section{Tumor xenograft study}

6 week-old female BALB/c nude mice were obtained from Beijing Vital River Laboratory Animal Technology Co., Ltd. (Beijing, China). All animal experimental procedures were performed according to the Institutional Guidelines and Animal Ordinance of the Department of Health, and approved by the Hong Kong Polytechnic University Animal Subjects Ethics Sub-committee.

Mice were inoculated with MDA-MB-231 cells $\left(5 \times 10^{6}\right)$ in the fourth mammary fat pad. After two weeks inoculation, the average tumor volume reach to $70 \mathrm{~mm}^{3}$, mice were randomly divided into four groups ( 6 mice per group), and treated by oral gavage with 25 or $50 \mathrm{mg} / \mathrm{kg} /$ day of $\mathrm{AD}$, or an equivalent amount of solvent $(0.5 \% \mathrm{CMCNa}, 1 \%$ Tween-80, 10 $\mathrm{mL} / \mathrm{kg}$ ) as a negative control (vehicle group). A positive control group received $10 \mathrm{mg} / \mathrm{kg}$ of docetaxel once a week by intraperitoneal (i.p.) injection. Body weight and tumor size were measured every two or three days during the experimental period. Tumor size was monitored using calipers and tumor volumes were calculated using the formula volume $=($ length $\times$ width $\left.^{2}\right) / 2$. Treatment duration was 22 days, and the average tumor volume of the vehicle group reached $800 \mathrm{~mm}^{3}$ during that time. At the experimental endpoint, all mice were sacrificed and their tumors and vital organs were harvested and weighed.

Formalin fixed tumor tissues were embedded with paraffin, and $5 \mu \mathrm{m}$ sections were cut serially and stained with hematoxylin and eosin (H\&E). Immunohistochemical detection of $\mathrm{Ki} 67$ was performed using the SPlink Detection Kit (ZSGB-BIO, Beijing, China), following manufacturer's protocols. In briefly, sections were deparaffinized and rehydrated, antigen were retrieved by heating in citric acid solution. Then, sections were incubated with $\mathrm{H}_{2} \mathrm{O}_{2}$ to quench endogenous peroxidase activity, blocked with normal goat serum working solution, incubated with primary antibody against Ki67 (1:1000

dilution; Proteintech, Wuhan, Hubei, China) at $4^{\circ} \mathrm{C}$ overnight. After rinsing three times with PBS, sections were incubated with biotin-labeled goat anti-rabbit IgG and horseradish peroxidase-labeled streptavidin. A final incubation in $\mathrm{DAB}$ chromogenic reagent was performed for visualization. All sections were stained with hematoxylin, dehydrated, cleared, coverslipped and photographed.

\section{Statistical analysis}

All data are presented as means \pm SEM and analysed using one-way analysis of variance (ANOVA) followed by LSD post hoc or Dunnett's tests. Differences were considered to be statistically significant at $\mathrm{P}<0.05$.

\section{Results}

\section{AD inhibited the cell proliferation of breast cancer cells}

The anti-proliferative effects of $\mathrm{AD}$ in breast cancer cells were examined by MTT assay in two TNBC cell lines, MDA-MB-231 and MDA-MB-468, as well as the ER and PR positive cell line MCF7. Results showed that AD exhibited anti-cancer activity in a dose- and time-dependent manner (Table 1, Figure 2). $\mathrm{IC}_{50}$ values of $\mathrm{AD}$ in MDA-MB-231, MDA-MB-468, and MCF7 were 12.04, 9.507, and $15.19 \mu \mathrm{M}$ for $24 \mathrm{~h}$ treatment, 5.211, 3.405, and $9.083 \mu \mathrm{M}$ for $48 \mathrm{~h}$ treatment, and 3.258, 2.515, and $8.909 \mu \mathrm{M}$ for $72 \mathrm{~h}$ treatment, respectively. The inhibitory effects of $\mathrm{AD}$ in the TNBC cell lines MDA-MB-231 and MDA-MB-468 were superior to the non-TNBC cell line MCF7 after 48 and $72 \mathrm{~h}$ treatments.

Table 1. IC 50 values of $A D$ in human breast cancer cell lines $(\mu M)$

\begin{tabular}{llll}
\hline Cell line & $24 \mathrm{~h}$ & $48 \mathrm{~h}$ & $72 \mathrm{~h}$ \\
\hline MDA-MB-231 & 12.04 & 5.211 & 3.258 \\
MDA-MB-468 & 9.507 & 3.405 & 2.515 \\
MCF7 & 15.19 & 9.083 & 8.909 \\
\hline
\end{tabular}

A

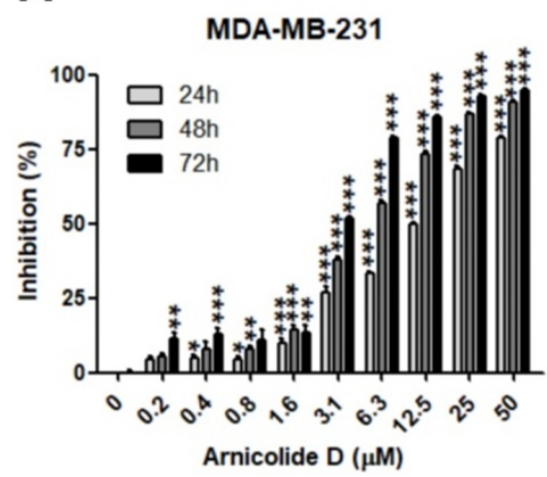

B

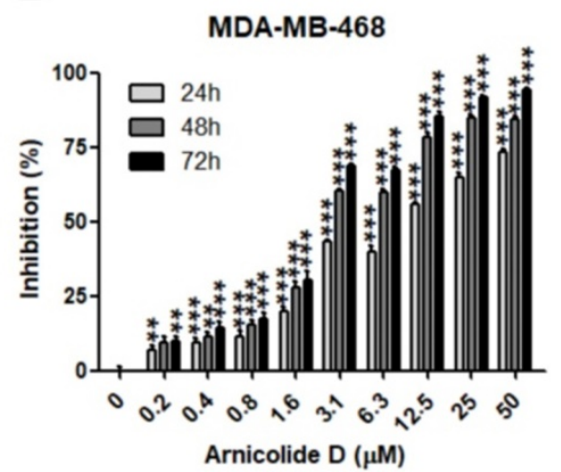

C

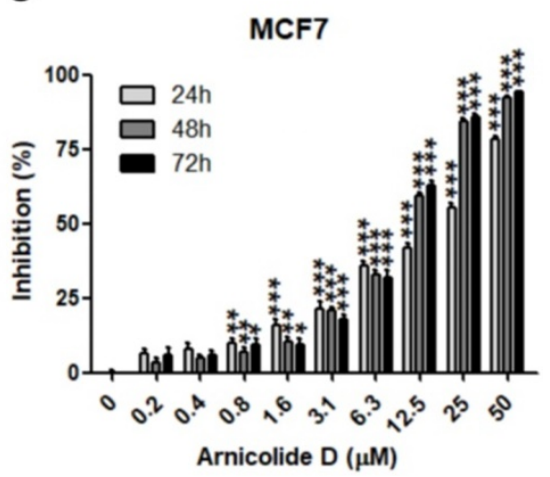

Figure 2. AD inhibited breast cancer cell proliferation. Cell viability of (A) MDA-MB-231, (B) MDA-MB-468, and (C) MCF7 was measured by MTT assay after AD treatment at the indicated concentrations for 24,48 and $72 \mathrm{~h}$. Data are presented as means $\pm \mathrm{SEM}$ from three independent experiments. $* \mathrm{P}<0.05$, $* * \mathrm{P}<0.01$, $* * * \mathrm{P}<0.001$, compared to control. 

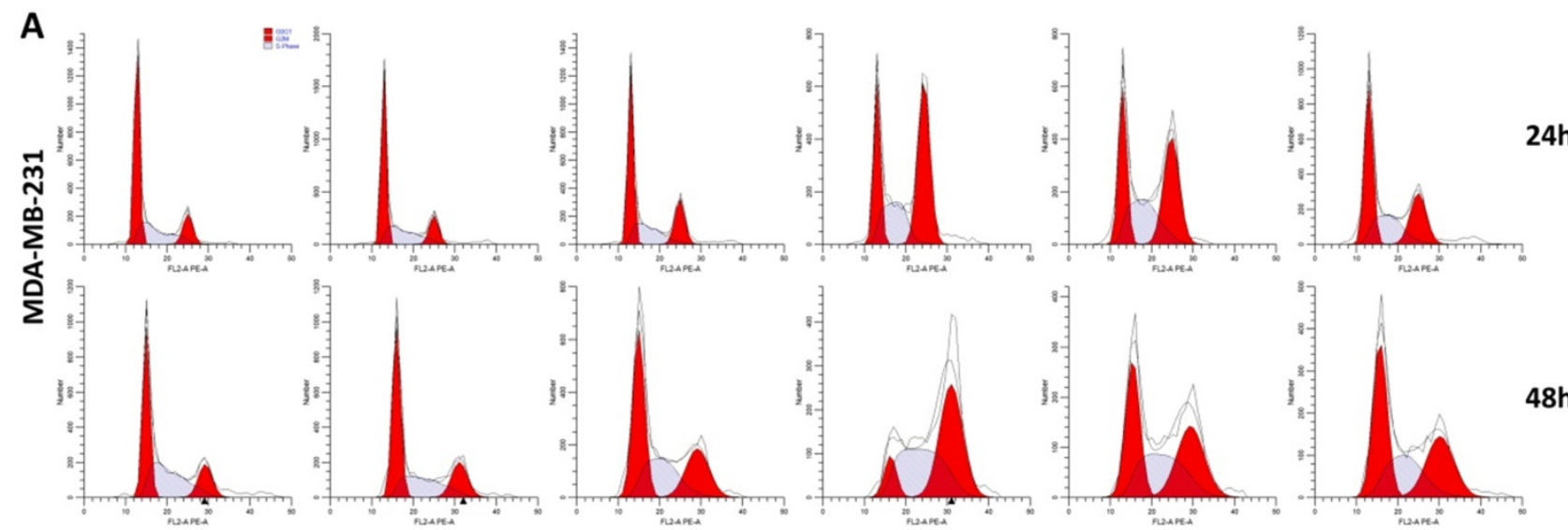

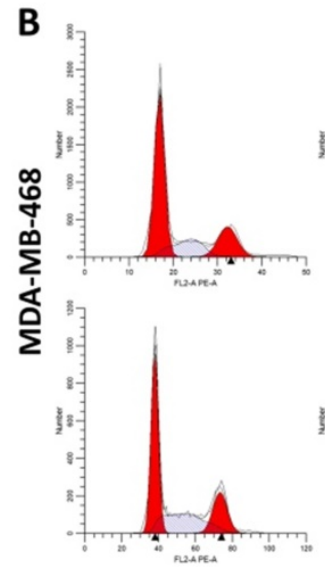

0
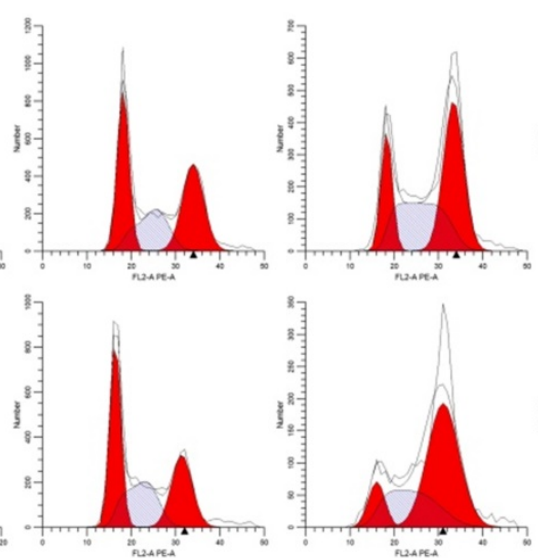

1.25

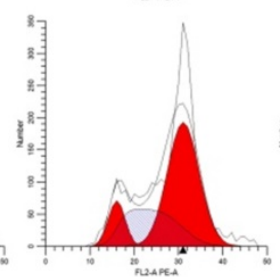

2.5
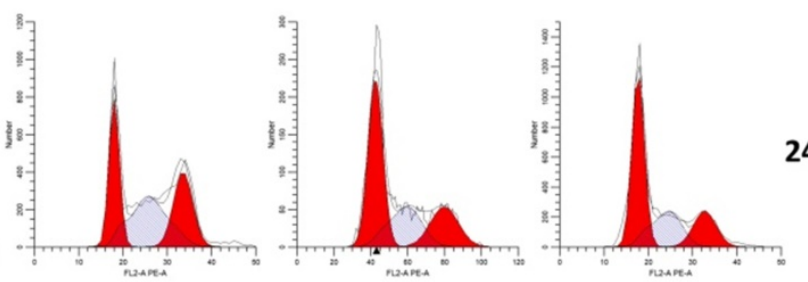

24h

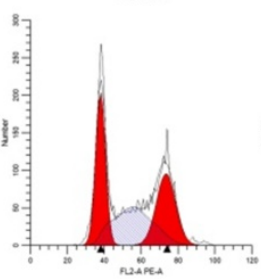

5

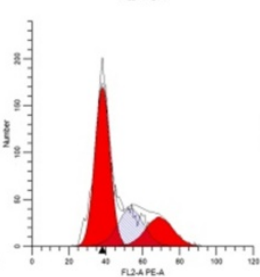

10

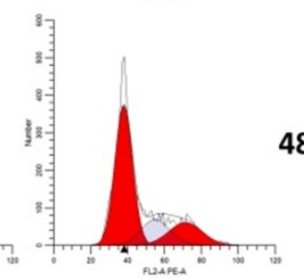

20

Figure 3. AD induced cell cycle arrest in TNBC. (A) MDA-MB-231 and (B) MDA-MB-468 cells were stained with PI and the cell cycle analyzed by flow cytometry.

\section{AD induced cell cycle arrest in TNBC cells}

To elucidate the underlying mechanisms of AD-induced inhibition, cell-cycle distribution patterns were analysed using flow cytometry in MDA-MB-231 and MDA-MB-468. As shown in Figure $3 \mathrm{~A}$, the proportion of MDA-MB-231 cells in the G2/M phase was significantly increased after $5 \mu \mathrm{M}$ AD treatment for both 24 and $48 \mathrm{~h}$, whereas higher doses of AD exhibited weaker inhibition of the cell cycle. Similarly, AD significantly increased MDA-MB-468 cell cycle arrest at the G2/M phase at a concentration of $2.5 \mu \mathrm{M}$ (Figure 3B). These results suggested that the anti-proliferative effect of $\mathrm{AD}$ may be through cell cycle arrest, combined with other mechanisms at higher doses.

\section{AD induced apoptosis in TNBC cells}

Apoptosis is another important factor contributing to cell proliferation. Thus, we measured apoptosis in TNBC cells with flow cytometry after AnnexinV-FITC/PI staining. Results showed that AD significantly increased the proportion of apoptotic cells at doses of 10 and $20 \mu \mathrm{M}$ (Figure 4). In
MDA-MB-231, the percentage of Annexin V positive cells increased by $27.93 \%$, and $29.37 \%$ after $24 \mathrm{~h}$ treatment, and 32.43\%, and $42.63 \%$ after $48 \mathrm{~h}$ treatment, respectively. In MDA-MB-468, the percentage of apoptotic cells increased by $22.68 \%$, and $36.68 \%$ after $24 \mathrm{~h}$ treatment, and $39.21 \%$, and $81.22 \%$ after $48 \mathrm{~h}$ treatment, respectively. These results indicated that low doses of AD led to cell cycle arrest, and as concentrations increased, eventually apoptosis.

To observe changes in the nuclear morphology of MDA-MB-231 and MDA-MB-468 cells, cells were photographed under an inverted fluorescence microscope after staining with Hoechst 33342. As shown in Figure 5, distinct chromatin condensation and formation of apoptotic bodies was observed after $24 \mathrm{~h}$ treatment of AD.

\section{AD inhibited cell migration in MDA-MB-231 cells}

Due to the highly invasive characteristic of TNBC, using the wound-healing assay, we investigated the inhibitive efficacy of $\mathrm{AD}$ on invasion in MDA-MB-231 cells. Results demonstrated that AD 
significantly suppressed cell migration at doses of 2.5, 5 , and $10 \mu \mathrm{M}$ after 24 and $48 \mathrm{~h}$ of treatment (Figure 6).

\section{AD inhibited Akt/mTOR signaling in TNBC cells}

Activation of the PI3K/Akt/mTOR signaling pathway plays a significant role in controlling cell growth, proliferation and metastasis, and has been identified as an important potential therapeutic target

A
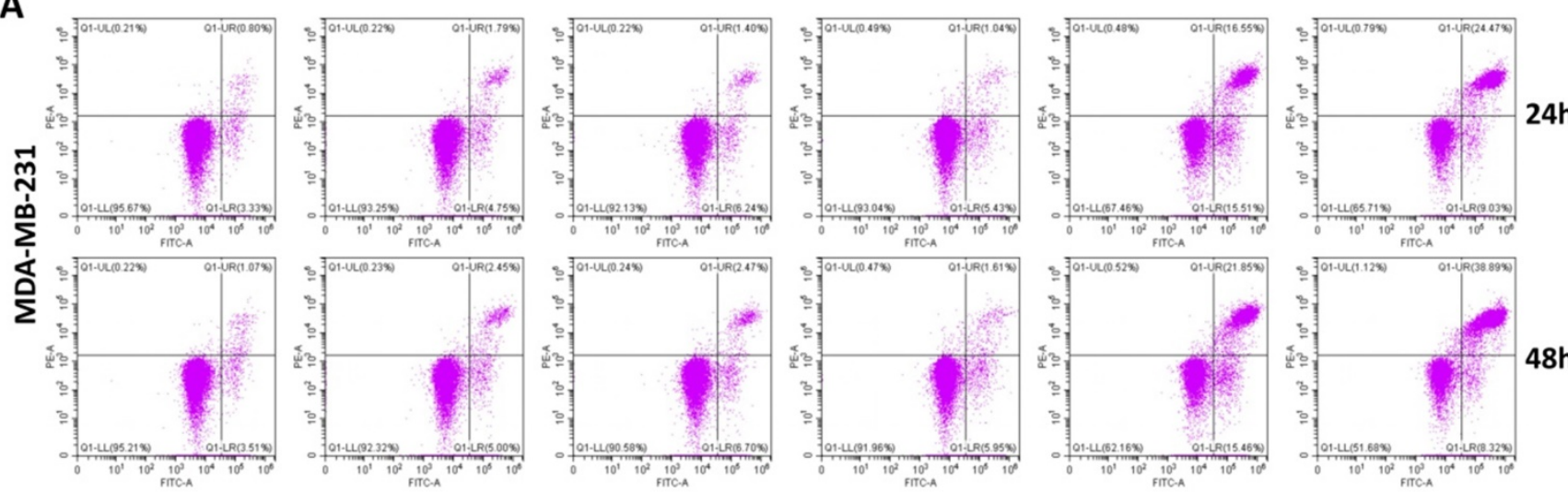

B

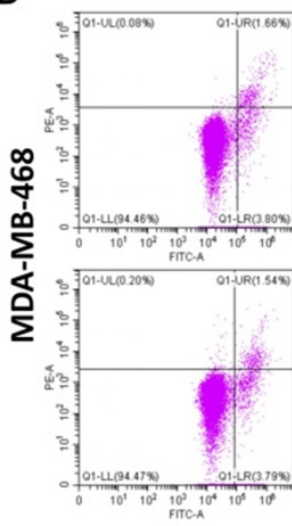

0
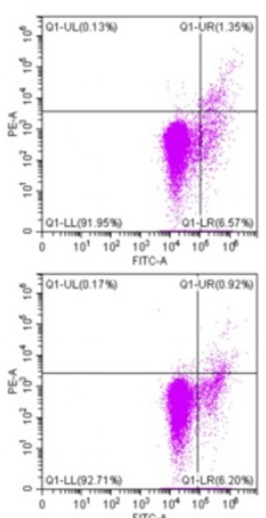

1.25

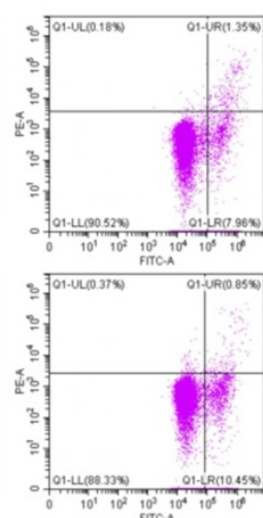

2.5

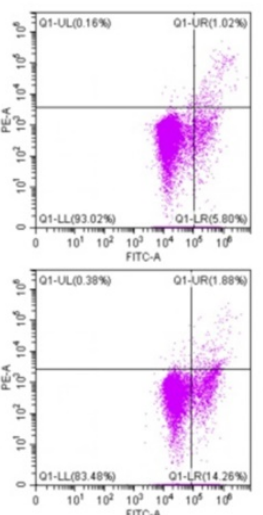

5

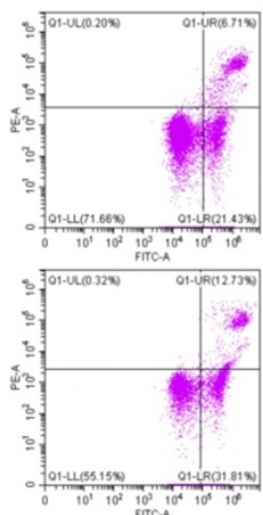

10

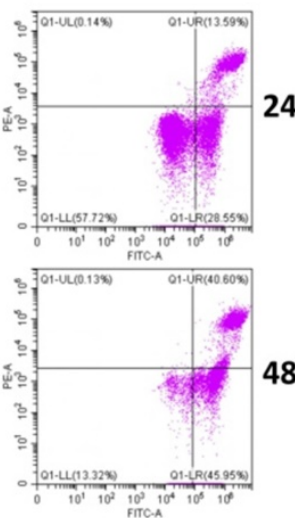

$A D(\mu M)$

Figure 4. AD induced apoptosis in TNBC. (A) MDA-MB-231 and (B) MDA-MB-468 were treated with AD, stained with AnnexinV-FITC/PI, and cell apoptosis analyzed by flow cytometry.
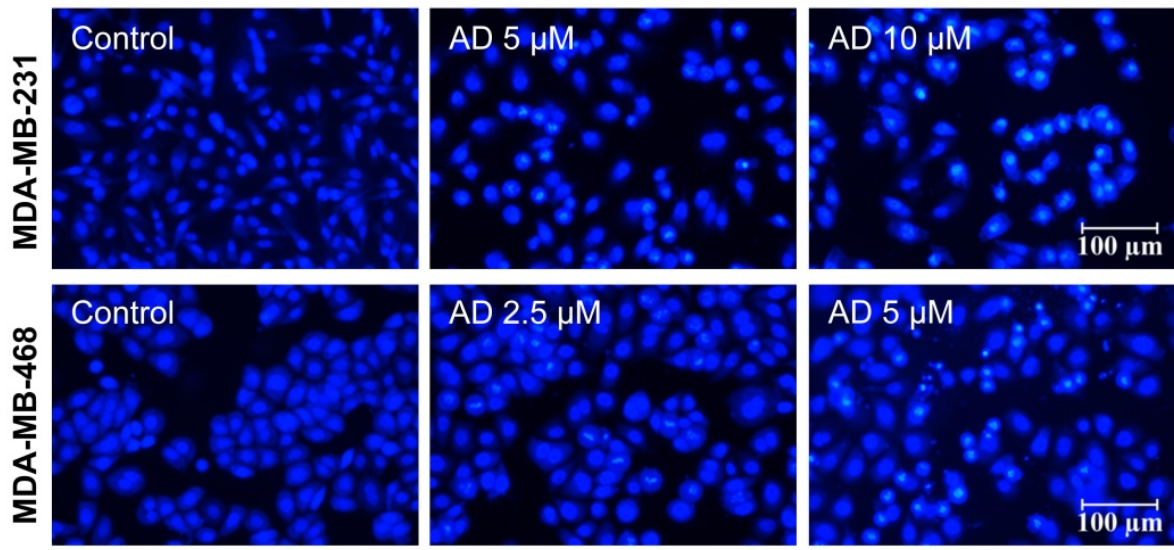

Figure 5. AD induced apoptotic body formation. MDA-MB-231 and MDA-MB-468 cells were treated with AD for 24 h, stained with Hoechst 33342 , and nuclear morphology was photographed using an inverted fluorescence microscope (200x). Scale bar $100 \mu \mathrm{m}$. 
A

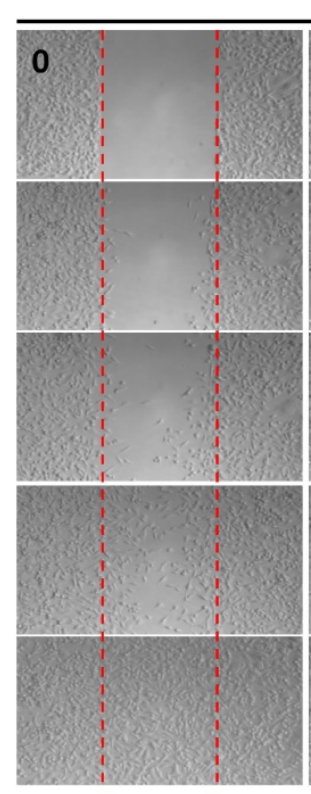

$A D(\mu M)$

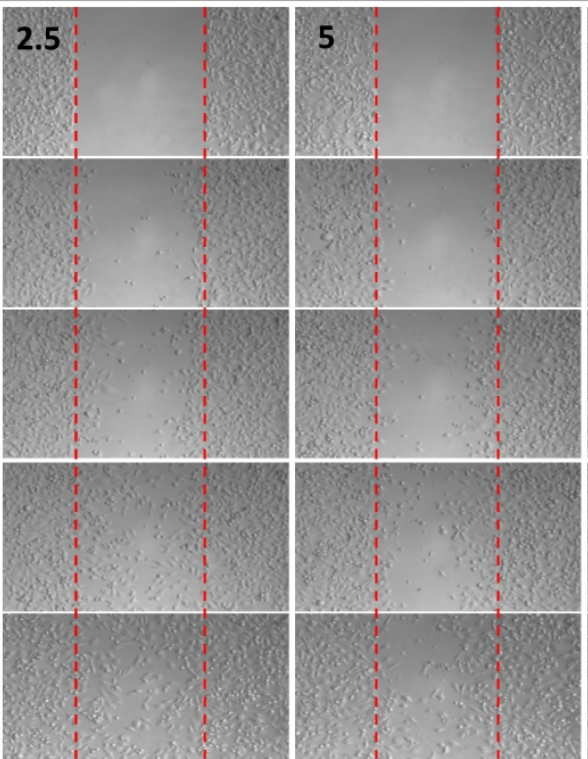

B
Batimastat $10 \mu \mathrm{M}$
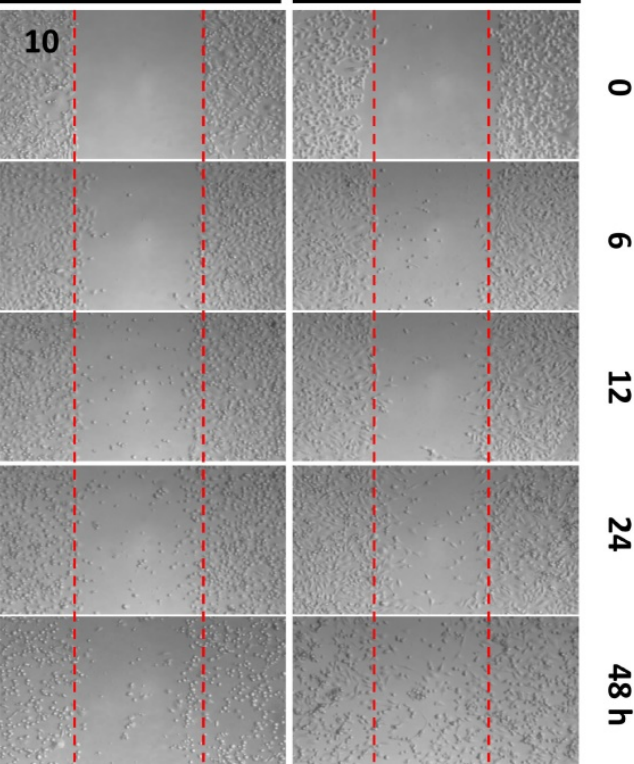

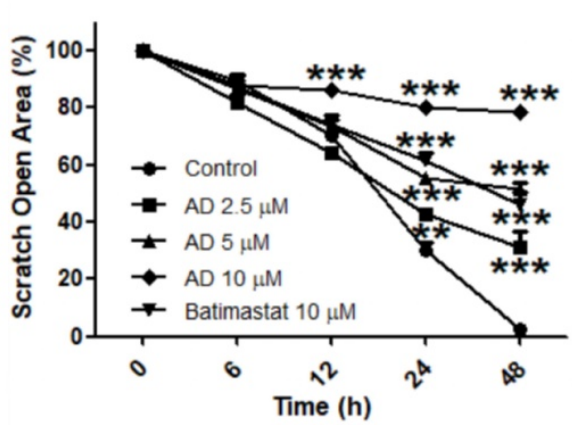

Figure 6. AD inhibited cell migration in MDA-MB-231 cells. (A) Cell migration was measured by wound-healing assay. (B) Quantification of scratch open area. Data are present as means \pm SEM from three independent experiments. $* \mathrm{P}<0.05$, $* * \mathrm{P}<0.01$, $* * * \mathrm{P}<0.001$, compared to control.

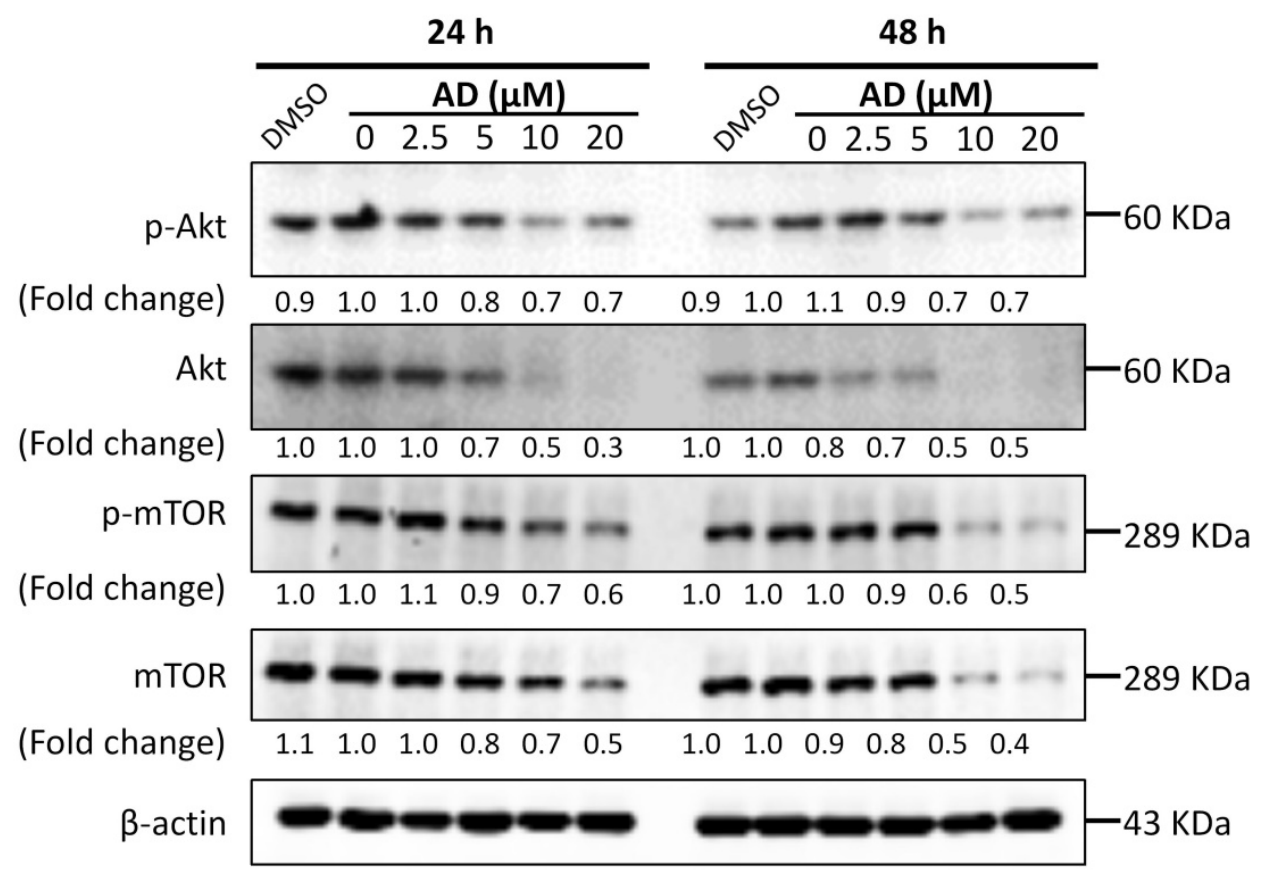

Figure 7. AD inhibited Akt/mTOR signaling in TNBC cells. MDA-MB-231 cells were treated with AD for 24 or $48 \mathrm{~h}$, collected and immunoblotted with the indicated antibodies. $\beta$-actin was used as an internal control. 


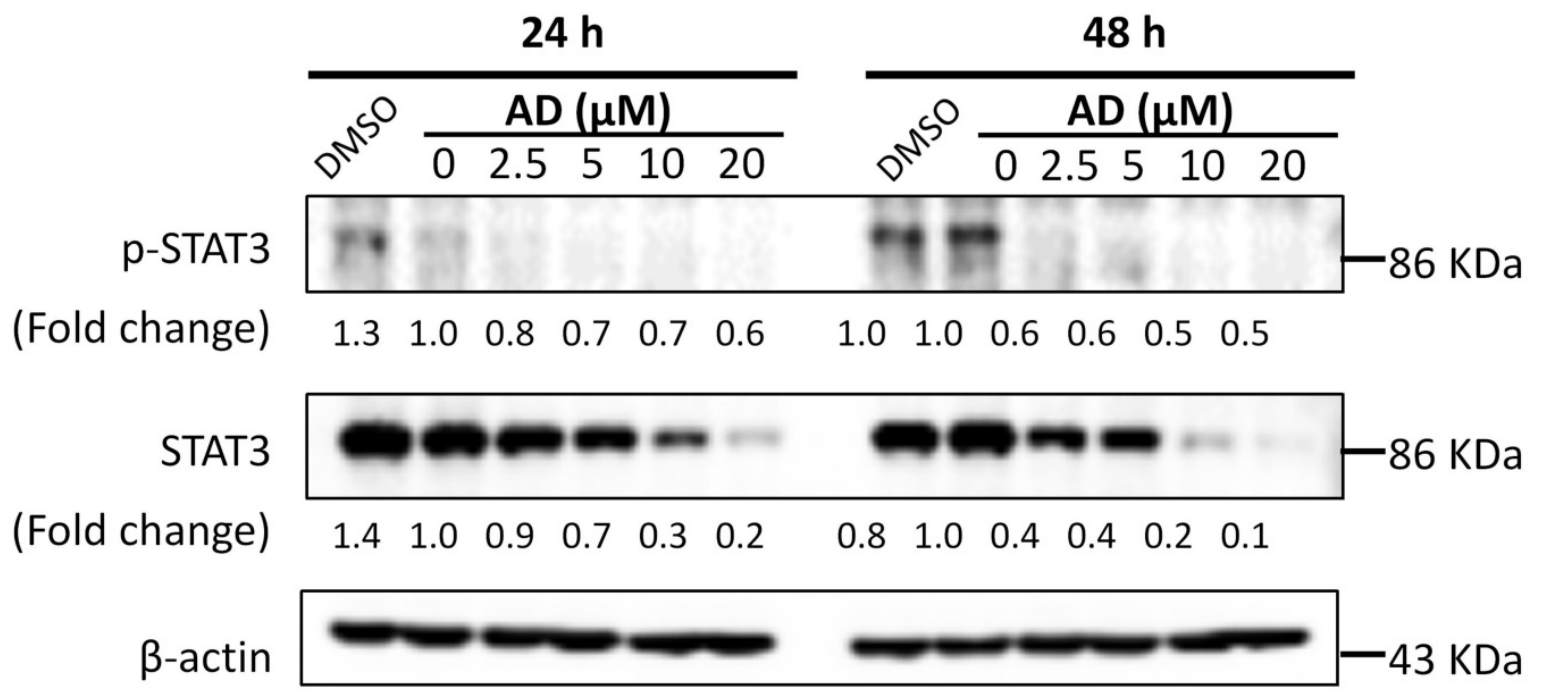

Figure 8. AD inhibited STAT3 signaling in TNBC cells. MDA-MB-231 cells were treated with AD for 24 or 48 h, collected and immunoblotted with the indicated antibodies. $\beta$-actin was used as an internal control.

\section{AD inhibited STAT3 signaling in TNBC cells}

STAT3 is an important transcription factor. Its overexpression and constitutive activation play a key role in the progression, proliferation and metastasis of breast cancer [14]. Therefore, expression levels of STAT3 and p-STAT3 were examined by Westernblotting after AD treatment. As shown in Figure 8, AD significantly downregulated the expression of STAT3 and p-STAT3 in a dose-dependent manner in MDAMB-231 cells.

\section{AD inhibited in vivo MDA-MB-231 xenograft tumor growth}

Based on the above results, we employed an orthotopic xenograft mouse model to investigate the in vivo anti-cancer effect of $\mathrm{AD}$. Mice received oral treatment with $\mathrm{AD}$ at a low $(25 \mathrm{mg} / \mathrm{kg})$ or high $(50$ $\mathrm{mg} / \mathrm{kg}$ ) doses, or i.p. injection of docetaxel as a positive control. At day 22, average tumor volumes were reduced by $35.1 \%$ (AD $25 \mathrm{mg} / \mathrm{kg}$ ), 49.5\% (AD 50 $\mathrm{mg} / \mathrm{kg}$ ), and $61.7 \%$ (docetaxel) when compared to vehicle control group (Figure 9A-B). Average tumor weights were reduced by $24.7 \%$ (AD $25 \mathrm{mg} / \mathrm{kg}$ ), 41.0\% (AD $50 \mathrm{mg} / \mathrm{kg}$ ) and $60.4 \%$ (docetaxel) when compared to control (Figure 9D). During the experimental period, AD did not cause significant body weight loss (Figure 9C).

To assess the changes in tumor morphology, the tumor sections were stained with H\&E. Similar to the docetaxel-treated group, the density of tumor cells in AD-treated group were notably decreased when compared to the vehicle group (Figure 10). We then further, examined the expression of cell proliferation marker Ki67. As shown in Figure 10, AD inhibited the expression of Ki67 when compared to the vehicle group. The above results indicated that $\mathrm{AD}$ exhibited in vivo anti-TNBC effects.

\section{Discussion}

TNBC exhibits a more aggressive phenotype and poorer prognosis than other breast cancer subtypes [4]. Natural products are a rich source for drug discovery of compounds to combat TNBC. In this study, we demonstrated that AD suppressed TNBC growth, in vitro and in vivo. AD exhibited inhibitory effects on the TNBC cell lines MDA-MB-231 and MDA-MB-468, and with a higher efficacy than in the non-TNBC cell line MCF7. Furthermore, oral administration of AD effectively inhibited xenograft tumor growth in vivo without apparent side effects.

Mitotic catastrophe and apoptosis are considered as two major types of cell death in cancer therapy [15]. In this study, we found that $\mathrm{AD}$ exhibited different effects at various concentrations. AD induced G2/M cell cycle arrest in MDA-MB-231 and MDA-MB-468 cells, while at higher doses, AD also exhibited significant apoptosis-inducing effects.

The PI3K/AKT/mTOR pathway regulates cell proliferation and survival, and is over-activated in more than $60 \%$ of TNBC patients [16]. In addition, pmTOR expression is associated with poor prognosis in early-stage TNBC [17]. In recent years, the PI3K/Akt/ mTOR pathway has been considered as a therapeutic target for novel drug discovery and several pathway inhibitors such as PQR309, Ipatasertib, AZD5363, and temsirolimus are being evaluated in clinical trials [18]. In this study, we found that $\mathrm{AD}$ downregulated the expression of AKT and mTOR in TNBC cells. Our results suggested that the inhibition of PI3K/ AKT/mTOR pathway may be a potential mechanism for the anti-TNBC activity of AD. 

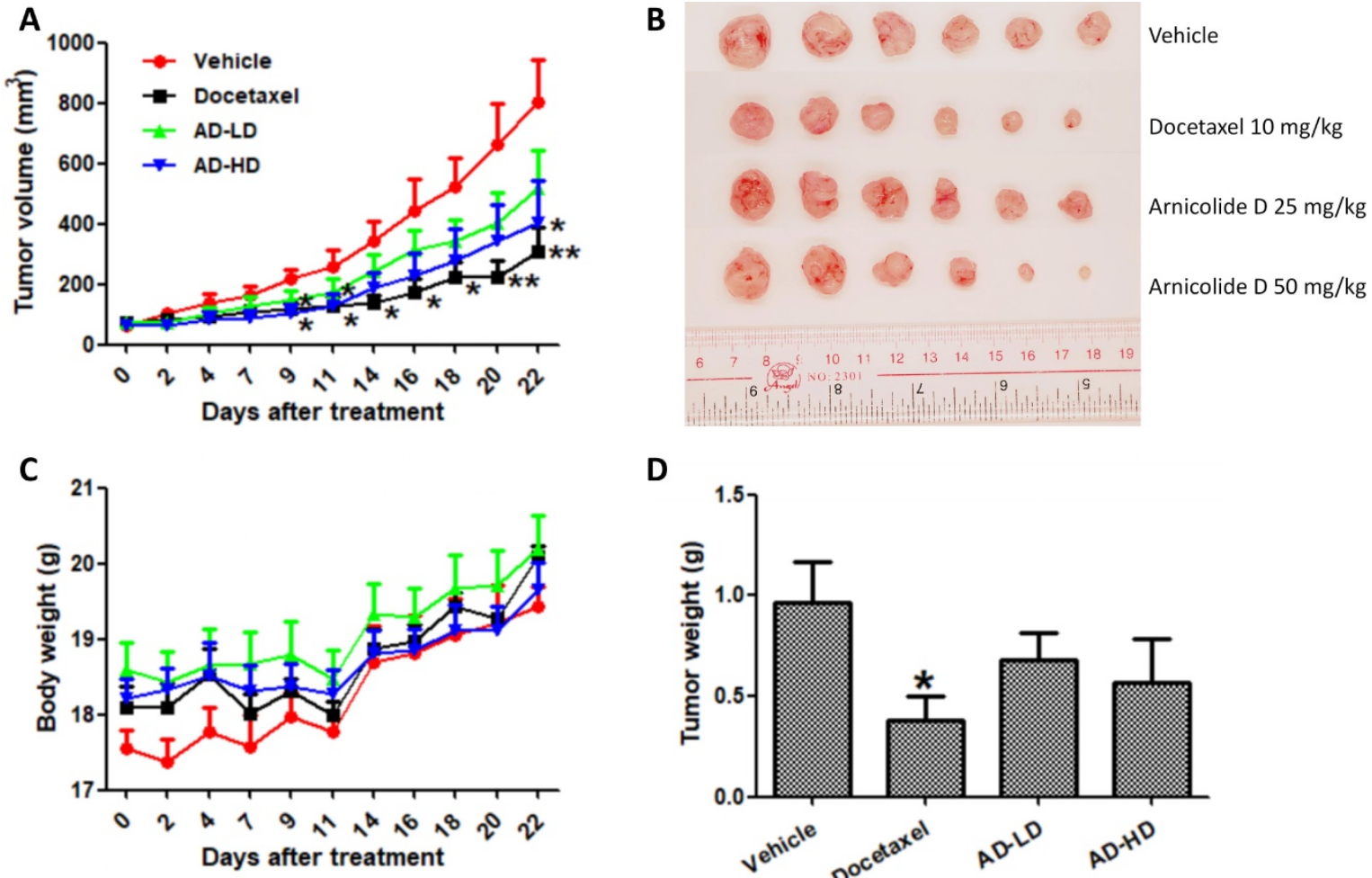

D

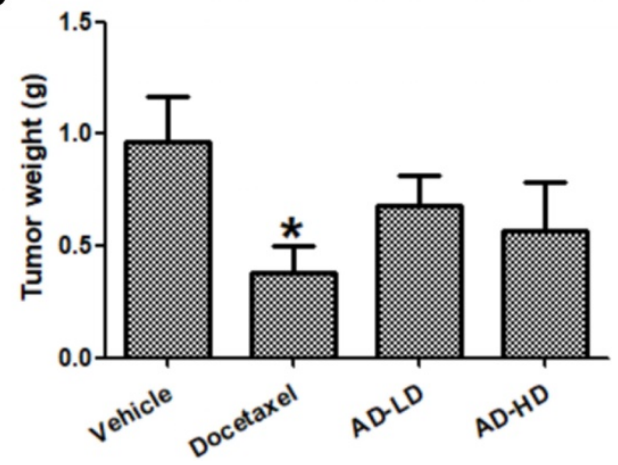

Figure 9. $A D$ inhibited in vivo MDA-MB-231 xenograft tumor growth. Female BALB/c nude mice were inoculated with MDA-MB-231 cells in their mammary fat pads. Two weeks later, mice were randomized into four groups and received treatment with vehicle ( $0.5 \% \mathrm{CMCNa}$, $1 \%$ Tween-80), AD-LD (AD $25 \mathrm{mg} / \mathrm{kg})$, or AD-HD (AD $50 \mathrm{mg} / \mathrm{kg}$ ) daily via oral adminstration, or docetaxel $(10 \mathrm{mg} / \mathrm{kg}$ ) once a week via i.p. injection. The treatment period lasted for 22 days after which all mice were sacrificed. (A) Tumor volumes of each group throughout the experimental period. (B) Images of tumors at experimental endpoint. (C) Mouse body weights throughout the experimental period. (D) Tumor weights at experimental endpoint. Data are expressed as means \pm SEM. $* P<0.05$, **P $<0.01$, compared to the vehicle group.

A

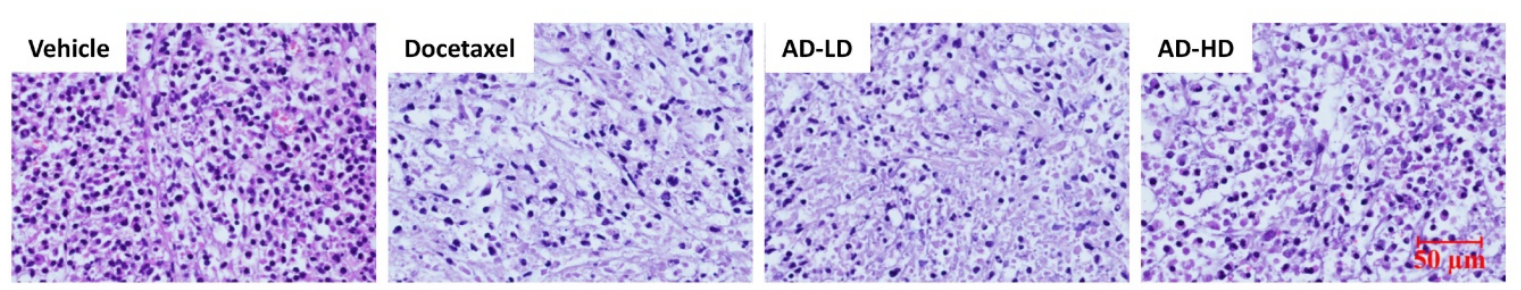

B

Ki67

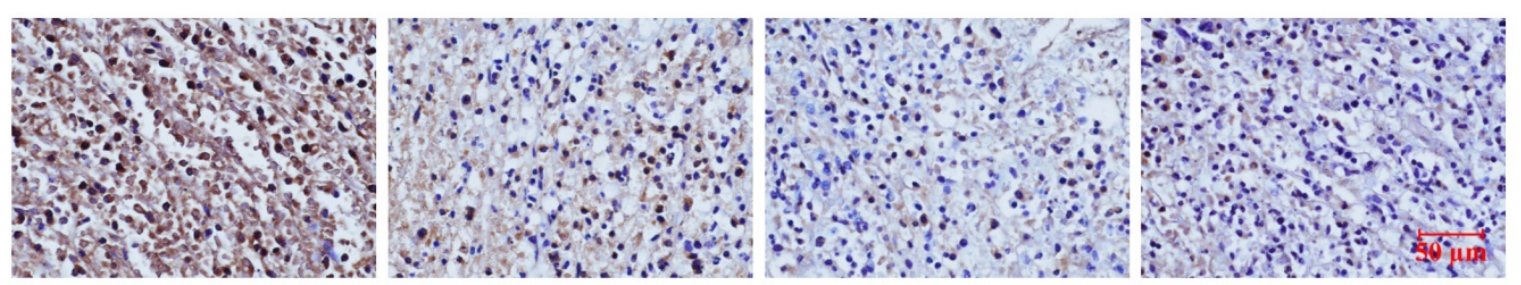

C

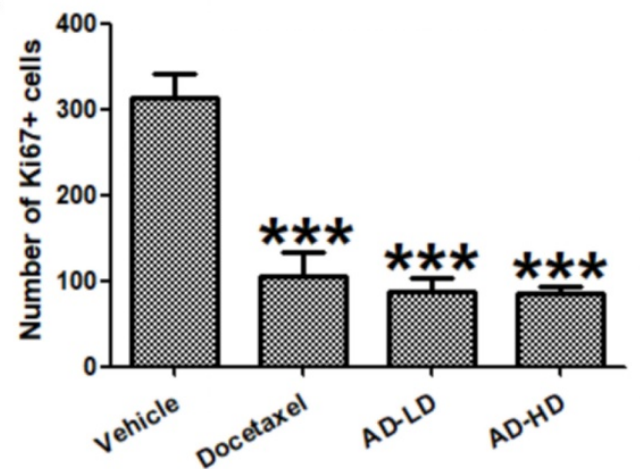

Figure 10. AD inhibited in vivo cancer cell proliferation. Tumors were stained with (A) H\&E and (B) Ki67. Representative images are shown (200x). Scale bar, $50 \mu m$. (C) Number of Ki67 positive cells are expressed as Means \pm SEM. $* * * P<0.001$, compared to the vehicle group. 
STAT3 is constitutively activated in more than $40 \%$ of breast cancers and is associated with initiation, progression, metastasis, chemoresistance, and immune evasion of TNBC $[19,20]$. Several natural sesquiterpene lactones, such as alantolactone, parthenolide and dehydrocostuslactone, exhibit antiTNBC effects via downregulation of p-STAT3 expression [21]. In the present study, we found that AD inhibited the expression of STAT3 in MDA-MB231 cells, which implies that STAT3 may be involved in the AD-mediated inhibition of TNBC cells as well.

A prior clinical study has reported that the mTOR and JAK2/STAT3 pathways are over-activated in inflammatory and invasive ductal breast cancers after neo-adjuvant chemotherapy [22]. Hence, the combination of therapy against mTOR and STAT3 is a promising prospect for the treatment of triple negative breast cancer [23]. In this study, $\mathrm{AD}$ exhibited significant inhibition of the AKT/mTOR and STAT3 pathways, and may be a promising drug candidate against TNBC.

In conclusion, this study demonstrates for the first time that AD could inhibit TNBC cell growth both in vitro and in vivo, and the mechanism of these effects was associated or at least partially associated with the inhibition of the Akt/mTOR and STAT3 signaling pathways. Our findings suggest $\mathrm{AD}$ as a potential novel adjunctive therapeutic drug for TNBC.

\section{Acknowledgements}

This work was supported in part by the Basic Research Foundation of the Shenzhen Science and Technology Innovation Committee (JCYJ20160229173 844278), and the Health and Medical Research Fund, Food and Health Bureau, Hong Kong SAR (project no. 16172701) to WT.

\section{Author Contributions}

WT, SC, and DM contributed to the conceptualization and design of the study. ZQ, YL and $\mathrm{QB}$ conducted the experiments. ZQ and $\mathrm{YL}$ wrote the manuscript. WT and SC contributed to manuscript revision. All authors read and approved the final manuscript.

\section{Competing Interests}

The authors have declared that no competing interest exists.

\section{References}

1. Bray F, Ferlay J, Soerjomataram I, et al. Global cancer statistics 2018: GLOBOCAN estimates of incidence and mortality worldwide for 36 cancers in 185 countries. CA Cancer J Clin.2018; 68:394-424.

2. Garrido-Castro AC, Lin NU, Polyak K. Insights into Molecular Classifications of Triple-Negative Breast Cancer: Improving Patient Selection for Treatment. Cancer Discov.2019; 9:176-198.

3. Shastry M, Yardley DA. Updates in the treatment of basal/triple-negative breast cancer. Curr Opin Obstet Gynecol. 2013; 25:40-48.
4. Lee KL, Kuo YC, Ho YS, et al. Triple-Negative Breast Cancer: Current Understanding and Future Therapeutic Breakthrough Targeting Cancer Stemness. Cancers 2019; 11(9): 1334.

5. Rahman NA, Yazan LS, Wibowo A, et al. Induction of apoptosis and G2/M arrest by ampelopsin E from Drybalanops towards triple negative breast cancer cells, MDA-MB-231. BMC Complement Altern Med. 2016; 16: 354.

6. Feng T, Cao W, Shen $W$, et al. Arctigenin inhibits STAT3 and exhibits anticancer potential in human triple-negative breast cancer therapy. Oncotarget 2017; 8 (1): 329-344.

7. Kwak JH, Park JY, Lee D, et al. Inhibitory effects of ginseng sapogenins on the proliferation of triple negative breast cancer MDA-MB-231 cells. Bioorg Med Chem Lett. 2014; 10 (24): 5409-5412.

8. Yang B, Huang J, Xiang T, et al. Chrysin inhibits metastatic potential of human triple-negative breast cancer cells by modulating matrix metalloproteinase-10, epithelial to mesenchymal transition, and PI3K/Akt signaling pathway. J Appl Toxicol. 2014; 34 (1): 105-112.

9. Taylor RS, Towers GH. Antibacterial constituents of the Nepalese medicinal herb, Centipeda minima. Phytochemistry.1998; 47: 631-634.

10. Huang X, Awano $\mathrm{Y}$, Maeda E, et al. Cytotoxic activity of two natural sesquiterpene lactones, isobutyroylplenolin and arnicolide $\mathrm{D}$, on human colon cancer cell line HT-29. Nat Prod Res. 2014; 28: 914-916.

11. Zhu P, Zheng Z, Fu X, et al. Arnicolide D exerts anti-melanoma effects and inhibits the NF-KB pathway. Phytomedicine 2019; 64: 153065.

12. Liu R, Dow Chan B, Mok DK, et al. Arnicolide D, from the herb Centipeda minima, Is a Therapeutic Candidate against Nasopharyngeal Carcinoma. Molecules 2019; 24: 1908.

13. Ortega MA, Fraile-Martínez O, Asúnsolo Á, et al. Signal Transduction Pathways in Breast Cancer: The Important Role of PI3K/Akt/mTOR. J Oncol. 2020; 2020: 9258396.

14. Ma JH, Qin L, Li X. Role of STAT3 signaling pathway in breast cancer. Cell Commun Signal. 2020; 138 (11): 2570-2578.

15. Pérez-Tomás R. Molecular mechanisms of cancer cell death. Curr Pharm Des. 2010; 16:1-2.

16. Kalimutho M, Parsons $\mathrm{K}$, Mittal D, et al. Targeted Therapies for Triple-Negative Breast Cancer: Combating a Stubborn Disease. Trends Pharmacol Sci. 2015; 36: 822-846.

17. Ueng SH, Chen SC, Chang YS, et al. Phosphorylated mTOR expression correlates with poor outcome in early-stage triple negative breast carcinomas. Int J ClinExpPathol. 2012; 5: 806-813.

18. Khan MA, Jain VK, Rizwanullah M, et al. PI3K/AKT/mTOR pathway inhibitors in triple-negative breast cancer: a review on drug discovery and future challenges. Drug Discov Today 2019; 24:2181-2191.

19. Qin JJ, Yan L, Zhang J, et al. STAT3 as a potential therapeutic target in triple negative breast cancer: a systematic review. J Exp Clin Cancer Res. 2019; 38 (1): 195.

20. Craig DW, O'Shaughnessy JA, Kiefer JA, et al. Genome and transcriptome sequencing in prospective metastatic triple-negative breast cancer uncovers therapeutic vulnerabilities. Mol Cancer Ther. 2013; 12:104-116.

21. Butturini E, Carcereri de Prati A, Boriero D, et al. Natural Sesquiterpene Lactones Enhance Chemosensitivity of Tumor Cells through Redox Regulation of STAT3 Signaling. Oxid Med Cell Longev. 2019; 2019: 4568964.

22. Jhaveri K, Teplinsky E, Silvera D, et al. HyperactivatedmTOR and JAK2/STAT3 Pathways: Molecular Drivers and Potential Therapeutic Targets of Inflammatory and Invasive Ductal Breast Cancers After Neoadjuvant Chemotherapy. Clin Breast Cancer. 2016; 16: 113-122.

23. Vundavilli H, Datta A, Sima $C$, et al. Bayesian Inference Identifies Combination Therapeutic Targets in Breast Cancer. IEEE Trans Biomed Eng. 2019; 66: 2684-2692. 